\title{
Calculation of Some Integrals Arising in the Samara-Valencia Solution for Dry Flat Grinding
}

\author{
J. L. González-Santander \\ Universidad Católica de Valencia "San Vicente Mártir", 46001 Valencia, Spain \\ Correspondence should be addressed to J. L. González-Santander; martinez.gonzalez@ucv.es
}

Received 29 June 2014; Revised 25 November 2014; Accepted 12 February 2015

Academic Editor: Christopher Gunaseelan Jesudason

Copyright ( 2015 J. L. González-Santander. This is an open access article distributed under the Creative Commons Attribution License, which permits unrestricted use, distribution, and reproduction in any medium, provided the original work is properly cited.

In the framework of the Samara-Valencia solution for heat transfer in grinding, two nontabulated integrals involving Macdonald's function of zeroth order are calculated.

\section{Introduction}

Grinding is a machining process that consists in the material removal from a workpiece by an abrasive wheel that rotates at a high speed over its surface [1] (see Figure 1). This kind of machining is very common in industry, so any improvement represents a significant reduction in production costs. In grinding, almost all the energy is converted into heat which is concentrated in the contact zone between the wheel and the workpiece [2]. Thermal damage occurs when temperature surpasses the phase transformation of the surface material. For over 50 years, we can find an extensive literature about thermal damage in grinding [3-9]. Usually, in order to prevent thermal damage, liquid coolant is injected onto the workpiece surface, so power generation by friction is reduced and cooling by convection occurs [10].

Traditionally, most of the studies of dry grinding temperature are based on Jaeger's model $[11,12]$, where a heat source of constant strength sliding over the surface of a half-plane is considered. DesRuisseaux and Zerkle [13] extended Jaeger's model to include the effect of surface cooling. Andrews et al. [14] investigate the well posedness of mathematical models for heat transfer in dry grinding. More recently, the SamaraValencia solution [15] has been proposed. As in [16], this solution is assumed to be two-dimensional; see Figure 1. The differential equation that governs the heat transfer is the convective heat equation. Also, the heat flux profile entering the workpiece and the action of the coolant are considered in the boundary condition. In [15], this two-dimensional boundary-value problem is transformed into an integral equation that is useful for the numerical evaluation of the heat transfer in intermittent wet grinding [17]. However, in the case of dry grinding, this integral equation can be reduced to a two-dimensional integral [18]. The latter is called $T^{(0)}$ theorem.

This paper is written from the point of view in which an engineering problem provides a new mathematical result. In fact, this point of view is very classical. For instance, Euler was the first one to give a proof (although not rigorous) of the equality of mixed partial derivatives, when he was working in hydrodynamics [19]. Another example is the engineering problem of measuring the coastline, which initiates the study of fractals in mathematics [20]. In the framework of grinding, comparing Jaeger's solution with the Samara-Valencia one, a new representation of the Dirac delta [21] and two new nontabulated integrals [22] have been obtained recently. The scope of this paper is just to provide another two nontabulated integrals by using $T^{(0)}$ theorem.

This paper is organized as follows. Section 2 describes the Samara-Valencia solution and the $T^{(0)}$ theorem. Section 3 derives from $T^{(0)}$ theorem two integrals involving Macdonald's function of zeroth order that seem not to be found in the usual literature [23-25]. 


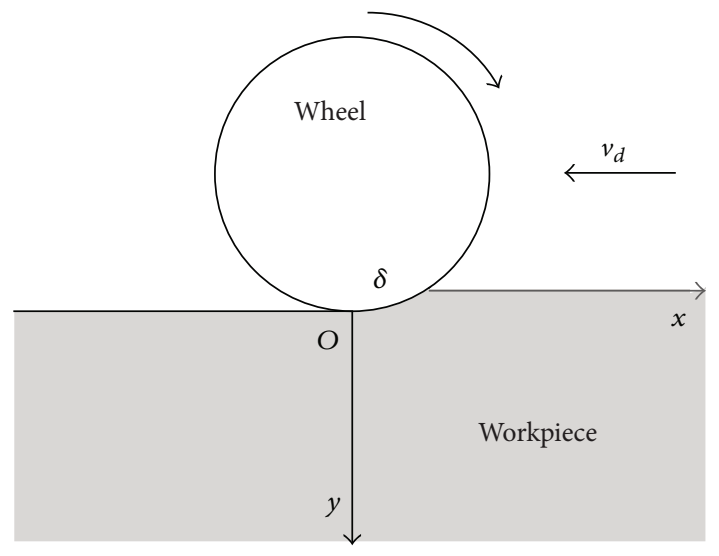

FIGURE 1: Two-dimensional modelling of flat grinding.

\section{Samara-Valencia Solution}

Figure 1 shows the setup assumed in the Samara-Valencia grinding model. The differential equation that governs the heat transfer is

$$
\begin{aligned}
\frac{\partial T(t, x, y)}{\partial t}= & k\left(\frac{\partial^{2} T(t, x, y)}{\partial x^{2}}+\frac{\partial^{2} T(t, x, y)}{\partial y^{2}}\right) \\
& -v_{d} \frac{\partial T(t, x, y)}{\partial x},
\end{aligned}
$$

where $T(t, x, y)$ is the rise temperature of the workpiece with respect to the room temperature, $k$ the thermal diffusivity, and $v_{d}$ the workpiece velocity with respect to the grinding wheel. According to Figure 1, the Cartesian coordinates $x$ and $y$ are referred to as the grinding wheel. The initial condition is

$$
T(0, x, y)=0,
$$

and the boundary condition is

$$
k_{0} \frac{\partial T(t, x, 0)}{\partial y}=b(t, x) T(t, x, 0)+d(t, x),
$$

where $k_{0}$ is the thermal conductivity of the workpiece, $b(t, x)$ is the heat transfer coefficient over the surface of the workpiece, and $d(t, x)$ is the heat flux entering the workpiece. Also $-\infty<x<\infty$ and $t, y \geq 0$. Physically speaking, $b(t, x)$ takes into account the convection that occurs over the workpiece surface due to the wheel and the lubricant fluid (in wet grinding); meanwhile $d(t, x)$ takes into account the heat that the wheel generates by friction over the workpiece surface. However, experiments suggest that the convective action of the wheel can be neglected, because, in dry grinding, Jaeger's model (where $b(t, x)$ is zero) is used to fit the temperature field inside the workpiece, considering a triangular heat flux profile for $d(t, x)$ [26]. In any case, the heat transfer in grinding is a complex process, so the set of (1)-(3) provides just a model, that is, an approximation to reality.

According to [15], the solution of the boundary-value problem (1)-(3) can be expressed as

$$
T(t, x, y)=T^{(0)}(t, x, y)+T^{(1)}(t, x, y),
$$

where we have defined

$$
\begin{aligned}
T^{(0)}(t, x, y) & \\
= & \frac{-1}{4 \pi k_{0}} \int_{0}^{t} \exp \left(\frac{-y^{2}}{4 k s}\right) \frac{d s}{s} \\
& \times \int_{-\infty}^{\infty} d\left(t-s, x^{\prime}\right) \exp \left(-\frac{\left(x^{\prime}-x-v_{d} s\right)^{2}}{4 k s}\right) d x^{\prime}, \\
T^{(1)}( & t, x, y) \\
= & \frac{1}{4 \pi} \int_{0}^{t} \exp \left(\frac{-y^{2}}{4 k s}\right) \frac{d s}{s} \int_{-\infty}^{\infty}\left(\frac{y}{2 k s}-\frac{b\left(t-s, x^{\prime}\right)}{k_{0}}\right) \\
& \times T\left(t-s, x^{\prime}, 0\right) \exp \left(-\frac{\left(x^{\prime}-x-v_{d} s\right)^{2}}{4 k s}\right) d x^{\prime} .
\end{aligned}
$$

As aforementioned, in dry grinding, we may assume that the heat transfer coefficient is zero:

$$
b(t, x)=0
$$

so (6) is reduced to

$$
\begin{aligned}
& T^{(1)}(t, x, y) \\
& =\frac{y}{8 \pi k} \int_{0}^{t} \exp \left(\frac{-y^{2}}{4 k s}\right) \frac{d s}{s^{2}} \\
& \quad \times \int_{-\infty}^{\infty} T\left(t-s, x^{\prime}, 0\right) \exp \left(-\frac{\left(x^{\prime}-x-v_{d} s\right)^{2}}{4 k s}\right) d x^{\prime} .
\end{aligned}
$$

Moreover, according to [18], we have

$$
T(t, x, y)=2 T^{(0)}(t, x, y)
$$

thus, taking into account (5), we obtain the temperature field in terms of a two-dimensional integral:

$$
\begin{aligned}
& T(t, x, y) \\
& =\frac{-1}{2 \pi k_{0}} \int_{0}^{t} \exp \left(\frac{-y^{2}}{4 k s}\right) \frac{d s}{s} \\
& \quad \times \int_{-\infty}^{\infty} d\left(t-s, x^{\prime}\right) \exp \left(-\frac{\left(x^{\prime}-x-v_{d} s\right)^{2}}{4 k s}\right) d x^{\prime} .
\end{aligned}
$$

The result given in (10) is called $T^{(0)}$ theorem. 


\section{Calculation of New Integrals from $T^{(0)}$ Theorem}

According to (4) and (9), we have

$$
T^{(0)}(t, x, y)=T^{(1)}(t, x, y) .
$$

Defining the function

$$
m(t, x, y)=\frac{1}{t} \exp \left(-\frac{\left(x+v_{d} t\right)^{2}+y^{2}}{4 k t}\right)
$$

and taking into account expressions (5) and (8) for $T^{(0)}$ and $T^{(1)}$, we may rewrite (11) as

$$
\begin{aligned}
T^{(0)} & (t, x, y) \\
= & \frac{-1}{4 \pi k_{0}} \int_{0}^{t} d s \int_{-\infty}^{\infty} d\left(t-s, x^{\prime}\right) m\left(s, x-x^{\prime}, y\right) d x^{\prime} \\
= & \frac{-1}{4 \pi} \int_{0}^{t} d s \int_{-\infty}^{\infty} T\left(t-s, x^{\prime}\right) \frac{\partial}{\partial y} m\left(s, x-x^{\prime}, y\right) d x^{\prime} .
\end{aligned}
$$

Using (9) and exchanging the integration order, we have

$$
\begin{aligned}
& T^{(0)}(t, x, y) \\
& \quad=\frac{-1}{4 \pi k_{0}} \int_{-\infty}^{\infty} d x^{\prime} \int_{0}^{t} d\left(t-s, x^{\prime}\right) m\left(s, x-x^{\prime}, y\right) d s \\
& \quad=\frac{-1}{2 \pi} \int_{-\infty}^{\infty} d x^{\prime} \int_{0}^{t} T^{(0)}\left(t-s, x^{\prime}, 0\right) \frac{\partial}{\partial y} m\left(s, x-x^{\prime}, y\right) d s .
\end{aligned}
$$

Applying the Laplace transform in (14),

$$
\tilde{f}(\tau)=\mathscr{L}[f(t)]=\int_{0}^{\infty} e^{-\tau t} f(t) d t,
$$

and taking into account the convolution theorem for this integral transform [24, Eq. 17.12.5]

$$
\mathscr{L}[f(t)] \mathscr{L}[g(t)]=\mathscr{L}\left[\int_{0}^{t} f(t-s) g(s) d s\right],
$$

(14) becomes

$$
\begin{aligned}
\widetilde{T}^{(0)} & (t, x, y) \\
= & \frac{-1}{4 \pi k_{0}} \int_{-\infty}^{\infty} \widetilde{d}\left(\tau, x^{\prime}\right) \widetilde{m}\left(\tau, x-x^{\prime}, y\right) d x^{\prime} \\
= & \frac{-1}{2 \pi} \int_{-\infty}^{\infty} \widetilde{T}^{(0)}\left(\tau, x^{\prime}, 0\right) \frac{\partial}{\partial y} \widetilde{m}\left(\tau, x-x^{\prime}, y\right) d x^{\prime} .
\end{aligned}
$$

Performing the Fourier transform in (17)

$$
\widehat{f}(w)=\mathscr{F}[f(x)]=\frac{1}{2 \pi} \int_{-\infty}^{\infty} e^{i w x} f(x) d x
$$

and taking into account the convolution theorem for this integral transform [27, Eq. 33.9]

$$
\mathscr{F}[f(x)] \mathscr{F}[g(x)]=\mathscr{F}\left[\frac{1}{2 \pi} \int_{-\infty}^{\infty} f\left(x^{\prime}\right) g\left(x-x^{\prime}\right) d x^{\prime}\right],
$$

we may rewrite (17) as

$$
\begin{aligned}
\widehat{\widetilde{T}}^{(0)}(\tau, w, y) & =\frac{-1}{2 k_{0}} \widehat{\tilde{\tilde{d}}}(\tau, w) \widehat{\bar{m}}(\tau, w, y) \\
& =-\widehat{\widetilde{T}}^{(0)}(\tau, w, y) \frac{\partial}{\partial y} \widehat{\widetilde{m}}(\tau, w, y) .
\end{aligned}
$$

Taking $y=0$ in (20) and substituting the result in (21), we arrive at

$$
\widehat{\bar{m}}(\tau, w, y)=-\widehat{\bar{m}}(\tau, w, 0) \frac{\partial}{\partial y} \widehat{\bar{m}}(\tau, w, y) .
$$

Solving the previous ODE (22), we get

$$
\widehat{\widetilde{m}}(\tau, w, y)=\widehat{\bar{m}}(\tau, w, 0) \exp \left(-\frac{y}{\widehat{\bar{m}}(\tau, w, 0)}\right), \quad y \geq 0,
$$

where, according to Figure $1 y \geq 0$. Later on, we will refer to (23) as the "master equation."

3.1. First Integral. First, let us perform the Laplace transform of the $m(t, x, y)$ function, defined in (12),

$$
\begin{aligned}
\widetilde{m}(\tau, x, y)= & \int_{0}^{\infty} e^{-\tau t} m(t, x, y) d t \\
= & \int_{0}^{\infty} \frac{e^{-\tau t}}{t} \exp \left(-\frac{\left(x+v_{d} t\right)^{2}+y^{2}}{4 k t}\right) d t \\
= & \exp \left(-\frac{x v_{d}}{2 k}\right) \\
& \cdot \int_{0}^{\infty} \exp \left(-\frac{x^{2}+y^{2}}{4 k t}-\left(\frac{v_{d}^{2}}{4 k}+\tau\right) t\right) \frac{d t}{t}
\end{aligned}
$$

Performing the change of variables $4 k s=\left(v_{d}^{2}+4 k \tau\right) t$ results in

$$
\begin{aligned}
\widetilde{m}(\tau, x, y)= & \exp \left(-\frac{x v_{d}}{2 k}\right) \\
& \cdot \int_{0}^{\infty} \exp \left(-\frac{\left(x^{2}+y^{2}\right)\left(v_{d}^{2}+4 k \tau\right)}{16 k^{2} s}-s\right) \frac{d s}{s} .
\end{aligned}
$$

Knowing that an integral representation of Macdonald's function of zeroth order is [28, Eq. 5.10.25]

$$
K_{0}(z)=\frac{1}{2} \int_{0}^{\infty} \exp \left(-\frac{z^{2}}{4 \sigma}-\sigma\right) \frac{d \sigma}{\sigma}, \quad|\arg z|<\frac{\pi}{4}
$$


we express (25) as

$$
\widetilde{m}(\tau, x, y)=2 \exp \left(-\frac{x v_{d}}{2 k}\right) K_{0}\left(\frac{\sqrt{\left(x^{2}+y^{2}\right)\left(v_{d}^{2}+4 k \tau\right)}}{2 k}\right) .
$$

Thus

$$
\widetilde{m}(\tau, x, 0)=2 \exp \left(-\frac{x v_{d}}{2 k}\right) K_{0}\left(|x| \frac{\sqrt{v_{d}^{2}+4 k \tau}}{2 k}\right) .
$$

On the one hand, applying the Fourier transform to (28), we have

$$
\begin{aligned}
\widehat{\widetilde{m}}(\tau, w, 0) & =\frac{1}{2 \pi} \int_{-\infty}^{\infty} \widetilde{m}(\tau, x, 0) e^{i w x} d x \\
& =\frac{1}{\pi} \int_{-\infty}^{\infty} \exp (-\alpha x) K_{0}(\beta|x|) d x,
\end{aligned}
$$

where we have set

$$
\begin{gathered}
\alpha=\frac{v_{d}}{2 k}-i w, \\
\beta=\frac{\sqrt{v_{d}^{2}+4 k \tau}}{2 k} .
\end{gathered}
$$

We may split (29) into two summands

$$
\begin{aligned}
\widehat{\bar{m}}(\tau, w, 0)= & \frac{1}{\pi} \int_{-\infty}^{0} \exp (-\alpha x) K_{0}(-\beta x) d x \\
& +\frac{1}{\pi} \int_{0}^{\infty} \exp (-\alpha x) K_{0}(\beta x) d x
\end{aligned}
$$

Changing $x$ by $-x$ in (32), we rewrite (29) as follows:

$$
\begin{aligned}
\widehat{\widetilde{m}}(\tau, w, 0) & =\frac{2}{\pi} \int_{0}^{\infty} \cosh (\alpha x) K_{0}(\beta x) d x \\
& =\frac{1}{\sqrt{\beta^{2}-\alpha^{2}}}, \quad \beta>|\alpha|,
\end{aligned}
$$

where we have applied the result given in Appendix (A.17).

On the other hand, applying the Fourier transform to (27) and taking into account the definitions of $\alpha$ and $\beta$ (30)-(31), we have

$$
\begin{aligned}
\widehat{\bar{m}}(\tau, w, y) & =\frac{1}{2 \pi} \int_{-\infty}^{\infty} \tilde{m}(\tau, x, y) e^{i w x} d x \\
& =\frac{1}{\pi} \int_{-\infty}^{\infty} \exp (-\alpha x) K_{0}\left(\beta \sqrt{x^{2}+y^{2}}\right) d x \\
& =\frac{2}{\pi} \int_{0}^{\infty} \cosh (\alpha x) K_{0}\left(\beta \sqrt{x^{2}+y^{2}}\right) d x
\end{aligned}
$$

Inserting now (33) and (34) into the "master equation" (23), we arrive at

$$
\begin{aligned}
\int_{0}^{\infty} \cosh (\alpha x) K_{0}\left(\beta \sqrt{x^{2}+y^{2}}\right) d x & \\
= & \frac{\pi \exp \left(-|y| \sqrt{\beta^{2}-\alpha^{2}}\right)}{2 \sqrt{\beta^{2}-\alpha^{2}}} \quad \beta>|\alpha|, y \in \mathbb{R},
\end{aligned}
$$

where we have extended the values of $y$, from $y \geq 0$ to $y \in \mathbb{R}$, by taking $|y|$ in the right hand side of (35). As far as the author's knowledge, (35) is not given in the literature. The equation most similar to (35) we have found in the the literature is [24, Eq. 6.677.5]

$$
\int_{0}^{\infty} \cos (\alpha x) K_{0}\left(\beta \sqrt{x^{2}+y^{2}}\right) d x=\frac{\pi \exp \left(-y \sqrt{\beta^{2}+\alpha^{2}}\right)}{2 \sqrt{\beta^{2}+\alpha^{2}}}
$$

$\operatorname{Re} \beta>0, \quad \operatorname{Re} y>0, \quad \alpha>0$.

See that, taking $\alpha=0$ and $\beta=1$ in (35), we have

$$
\int_{0}^{\infty} K_{0}\left(\sqrt{x^{2}+y^{2}}\right) d x=\frac{\pi}{2} e^{-|y|}, \quad y \in \mathbb{R} .
$$

Surprisingly, (37) is not reported in the usual literature.

3.2. Second Integral. Performing in the inverse Fourier transform in (22) by using the convolution theorem for Fourier transforms (19), we obtain

$$
\widetilde{m}(\tau, w, y)=\frac{-1}{2 \pi} \int_{-\infty}^{\infty} \widetilde{m}\left(\tau, x^{\prime}, 0\right) \frac{\partial}{\partial y} \widetilde{m}\left(\tau, x-x^{\prime}, y\right) d x^{\prime} .
$$

According to the definition of $\beta$ (31), we rewrite (27) as

$$
\widetilde{m}(\tau, w, y)=2 \exp \left(-\frac{x v_{d}}{2 k}\right) K_{0}\left(\beta \sqrt{x^{2}+y^{2}}\right) ;
$$

thus,

$$
\begin{gathered}
\widetilde{m}(\tau, w, 0)=2 K_{0}(\beta|x|) \\
\frac{\partial}{\partial y} \widetilde{m}(\tau, x, y)=-2 y \beta \exp \left(-\frac{x v_{d}}{2 k}\right) \frac{K_{1}\left(\beta \sqrt{x^{2}+y^{2}}\right)}{\sqrt{x^{2}+y^{2}}} .
\end{gathered}
$$

Substituting (39)-(40) into (38), we have

$$
\begin{aligned}
& K_{0}\left(\beta \sqrt{x^{2}+y^{2}}\right) \\
& =\frac{y \beta}{\pi} \int_{-\infty}^{\infty} K_{0}\left(\beta\left|x^{\prime}\right|\right) \frac{K_{1}\left(\beta \sqrt{\left(x-x^{\prime}\right)^{2}+y^{2}}\right)}{\sqrt{\left(x-x^{\prime}\right)^{2}+y^{2}}} d x^{\prime} .
\end{aligned}
$$


Taking $\xi=\beta x, v=\beta y$ and performing the change of variables $\xi^{\prime}=\beta x^{\prime}$, we arrive at

$$
K_{0}\left(\sqrt{\xi^{2}+v^{2}}\right)=\frac{v}{\pi} \int_{-\infty}^{\infty} K_{0}\left(\left|\xi^{\prime}\right|\right) \frac{K_{1}\left(\sqrt{\left(\xi-\xi^{\prime}\right)^{2}+v^{2}}\right)}{\sqrt{\left(\xi-\xi^{\prime}\right)^{2}+v^{2}}} d \xi^{\prime} .
$$

The integral given in (43) has been already calculated by using a complex integration contour [22]. However, we can get another new integral, integrating in (43) with respect to the $v$ variable between 0 and $\infty$, obtaining

$$
\begin{aligned}
\int_{0}^{\infty} K_{0}\left(\sqrt{\xi^{2}+v^{2}}\right) d v= & \frac{1}{\pi} \int_{-\infty}^{\infty} K_{0}\left(\left|\xi^{\prime}\right|\right) d \xi^{\prime} \\
& \times \int_{0}^{\infty} v \frac{K_{1}\left(\sqrt{\left(\xi-\xi^{\prime}\right)^{2}+v^{2}}\right)}{\sqrt{\left(\xi-\xi^{\prime}\right)^{2}+v^{2}}} d v .
\end{aligned}
$$

Taking into account (37) and (41), it turns out that

$$
\frac{\pi}{2} e^{-|\xi|}=\left.\frac{1}{\pi} \int_{-\infty}^{\infty} K_{0}\left(\left|\xi^{\prime}\right|\right) K_{0}\left(\sqrt{\left(\xi-\xi^{\prime}\right)^{2}+v^{2}}\right)\right|_{0} ^{\infty} d \xi^{\prime} .
$$

Since [28, Eq. 5.16.5]

$$
\lim _{x \rightarrow \infty} K_{0}(x)=\lim _{x \rightarrow \infty} \sqrt{\frac{\pi}{2 x}} e^{-x}=0,
$$

we obtain the following integral:

$$
\int_{-\infty}^{\infty} K_{0}\left(\left|\xi^{\prime}\right|\right) K_{0}\left(\left|\xi-\xi^{\prime}\right|\right) d \xi^{\prime}=\frac{\pi^{2}}{2} e^{-|\xi|} .
$$

As in (35), (47) seems not to be found in the usual literature. Notice that taking $\xi=0$ in (47) we recover the following integral given in the literature [24, Eq. 6.511.13]:

$$
\int_{0}^{\infty} K_{0}^{2}\left(\xi^{\prime}\right) d \xi^{\prime}=\frac{\pi^{2}}{4}
$$

\section{Appendix}

\section{Auxiliary Integral}

Let us calculate

$$
I=\int_{0}^{\infty} \cosh (\alpha x) K_{0}(\beta x) d x,
$$

splitting (A.1) into two summands

$$
I=\frac{I_{\alpha}+I_{-\alpha}}{2}
$$

where we have set

$$
I_{ \pm \alpha}=\int_{0}^{\infty} e^{ \pm \alpha x} K_{0}(\beta x) d x
$$

Despite the fact that we can calculate (A.3) by using [29, Eq. 4.13.5]

$$
\int_{0}^{\infty} e^{-t \cosh a} K_{0}(t) d t=\frac{a}{\sinh a}, \quad \operatorname{Re}(\cosh a)>-1,
$$

yielding the result

$$
I_{\alpha}=\frac{1}{\sqrt{\alpha^{2}-\beta^{2}}} \cosh ^{-1}\left(\frac{\mp \alpha}{\beta}\right), \quad \operatorname{Re}\left(\frac{-\alpha}{\beta}\right)>-1,
$$

here we provide the following derivation, because it leads to (A.14), which is more convenient for our purpose. Indeed, let us substitute in (A.3) the following integral representation of Macdonald's function of zeroth order [28, Eq. 5.10.23]:

$$
K_{0}(z)=\int_{0}^{\infty} \exp (-z \cosh t) d t, \quad \operatorname{Re} z>0,
$$

in (A.3) and let us exchange also the integration order, obtaining

$$
\begin{aligned}
I_{\alpha} & =\int_{0}^{\infty} d t \int_{0}^{\infty} \exp (-(\beta \cosh t \mp \alpha) x) d x \\
& =-\left.\int_{0}^{\infty} \frac{\exp (-(\beta \cosh t \mp \alpha) x)}{\beta \cosh t \mp \alpha}\right|_{0} ^{\infty} d t .
\end{aligned}
$$

Notice that if

$$
\beta \cosh t \mp \alpha>0 \text {, }
$$

then the inner integral of (A.7) converges. Since $1 \leq \cosh t<$ $\infty, \forall t \in(0, \infty)$, then (A.8) becomes

$$
\beta>\frac{ \pm \alpha}{\cosh t}> \pm \alpha \text {. }
$$

Therefore,

$$
I_{ \pm \alpha}=\int_{0}^{\infty} \frac{d t}{\beta \cosh t \mp \alpha}, \quad \beta>|\alpha| .
$$

We can calculate (A.10), performing the change of variables $z=\tanh t / 2$, so

$$
\begin{aligned}
& \cosh t=\frac{1+z^{2}}{1-z^{2}} \\
& d z=\frac{1-z^{2}}{2} d t
\end{aligned}
$$

Thus,

$$
\begin{aligned}
I_{ \pm \alpha} & =\frac{2}{\beta \mp \alpha} \int_{0}^{1} \frac{d z}{1+((\beta \pm \alpha) /(\beta \mp \alpha)) z^{2}} \\
& =\frac{2}{\sqrt{\beta^{2}-\alpha^{2}}} \tan ^{-1}\left(\sqrt{\frac{\beta \pm \alpha}{\beta \mp \alpha}}\right) .
\end{aligned}
$$


Remember that $\beta>|\alpha|$, so the radicals in (A.12) are positive. Applying (A.12) to (A.10) and taking into account the identity [24, Eq. 1.628.2]

$$
2 \tan ^{-1} x=\cos ^{-1}\left(\frac{1-x^{2}}{1+x^{2}}\right), \quad x \geq 0,
$$

we have

$$
I_{ \pm \alpha}=\frac{1}{\sqrt{\beta^{2}-\alpha^{2}}} \cos ^{-1}\left(\mp \frac{\alpha}{\beta}\right), \quad \beta>|\alpha| .
$$

Since [24, Eq. 1.623.1]

$$
\sin ^{-1} x+\cos ^{-1} x=\frac{\pi}{2}
$$

we may express (A.14) as

$$
\begin{gathered}
I_{\alpha}=\frac{\pi / 2+\sin ^{-1}(\alpha / \beta)}{\sqrt{\beta^{2}-\alpha^{2}}}, \\
I_{-\alpha}=\frac{\cos ^{-1}(\alpha / \beta)}{\sqrt{\beta^{2}-\alpha^{2}}} .
\end{gathered}
$$

Finally, substituting (A.16) in (A.2) and taking into account again (A.15), we obtain

$$
\int_{0}^{\infty} \cosh (\alpha x) K_{0}(\beta x) d x=\frac{\pi}{2 \sqrt{\beta^{2}-\alpha^{2}}}, \quad \beta>|\alpha| .
$$

\section{Conflict of Interests}

The author declares that there is no conflict of interests regarding the publication of this paper.

\section{References}

[1] S. Malkin, Grinding Technology: Theory and Application of Machining with Abrasives, Ellis Horwood, John Wiley \& Sons, 1989.

[2] J. O. Outwater and M. C. Shaw, "Surface temperature in grinding," Transactions of the ASME, vol. 74, pp. 73-86, 1952.

[3] R. S. Hahn, "The relation between grinding conditions and thermal damage in the workpiece," Transactions of the ASME, vol. 78, pp. 807-812, 1956.

[4] S. Malkin and R. B. Anderson, "Thermal aspects of grinding, part I: energy partition," Journal of Manufactured Science Engineering, vol. 96, no. 4, pp. 1177-1183, 1974.

[5] A. S. Lavine and B. F. von Turkovich, "Thermal aspects of grinding: the effects of heat generation at the shear planes," CIRP Annals-Manufacturing Technology, vol. 40, no. 1, pp. 343-345, 1991.

[6] A. S. Lavine, "An exact solution for surface temperature in down grinding," International Journal of Heat and Mass Transfer, vol. 43, no. 24, pp. 4447-4456, 2000.
[7] M. D. Demetriou and A. S. Lavine, "Thermal aspects of grinding: the case of upgrinding," Journal of Manufacturing Science and Engineering, vol. 122, no. 4, pp. 605-611, 2000.

[8] A. S. Lavine and T.-C. Jen, "Coupled heat transfer to workpiece, wheel, and fluid in grinding, and the occurrence of workpiece burn," International Journal of Heat and Mass Transfer, vol. 34, no. 4-5, pp. 983-992, 1991.

[9] A. S. Lavine and T. C. Jen, "Thermal aspects of grinding: heat transfer to workpiece, wheel, and fluid," Journal of Heat Transfer, vol. 113, no. 2, pp. 296-303, 1991.

[10] A. S. Lavine, "A simple model for convective cooling during the grinding process," Journal of Engineering for Industry, vol. 110, no. 1, pp. 1-6, 1988.

[11] J. C. Jaeger, "Moving sources of heat and the temperature at sliding contacts," Proceedings of Royal Society of New South Wales, vol. 76, pp. 204-224, 1942.

[12] H. S. Carslaw and J. C. Jaeger, Conduction of Heat in Solids, The Clarendon Press, Oxford, UK, 1959.

[13] N. R. DesRuisseaux and R. D. Zerkle, "Temperature in semiinfinite and cylindrical bodies subjected to moving heat surfaces and surface Cooling," Journal of Heat Transfer, vol. 92, pp. 456-464, 1970.

[14] K. T. Andrews, M. Shillor, and S. Wright, "A model for heat transfer in grinding," Nonlinear Analysis. Theory, Methods \& Applications, vol. 35, pp. 233-246, 1999.

[15] D. L. Skuratov, Y. L. Ratis, I. A. Selezneva, J. Pérez, P. F. de Córdoba, and J. F. Urchueguía, "Mathematical modelling and analytical solution for workpiece temperature in grinding," Applied Mathematical Modelling, vol. 31, no. 6, pp. 1039-1047, 2007.

[16] L. C. Zhang, T. Suto, H. Noguchi, and T. Waida, "An overview of applied mechanics in grinding," Manufacturing Review, vol. 5, no. 4, pp. 261-273, 1992.

[17] J. Pérez, S. Hoyas, D. L. Skuratov et al., "Heat transfer analysis of intermittent grinding processes," International Journal of Heat and Mass Transfer, vol. 51, no. 15-16, pp. 4132-4138, 2008.

[18] J. L. González-Santander, J. M. Valdés Placeres, and J. M. Isidro, "Exact solution for the time-dependent temperature field in dry grinding: application to segmental wheels," Mathematical Problems in Engineering, vol. 2011, Article ID 927876, 28 pages, 2011.

[19] T. J. Higgins, "A note on the history of mixed partial derivatives," Scripta Mathematica, vol. 7, pp. 59-62, 1940.

[20] B. B. Mandelbrot, "II. 5 how long is the coast of Britain?" in The Fractal Geometry of Nature, Macmillan, New York, NY, USA, 1977.

[21] J. L. González-Santander, J. Pérez, P. F. de Córdoba, and J. M. Isidro, "An analysis of the temperature field of the workpiece in dry continuous grinding," Journal of Engineering Mathematics, vol. 67, no. 3, pp. 165-174, 2010.

[22] J. L. G. Santander, P. Castañeda Porras, Y. L. Ratis, J. M. Isidro, and P. Fernández De Córdoba, "Calculation of some integrals arising in heat transfer in grinding," Mathematical Problems in Engineering, vol. 2010, Article ID 535801, 14 pages, 2010.

[23] M. Abramowitz and I. A. Stegun, Handbook of Mathematical Functions, Dover Publications, Washington, DC, USA, 1972.

[24] I. S. Gradshteyn and I. M. Ryzhik, Table of Integrals, Series and Products, Academic Press, New York, NY, USA, 7th edition, 2007.

[25] F. W. J. Olver, D.-W. Lozier, R. F. Boisvert, and C. W. Clark, NIST Handbook of Mathematical Functions, Cambridge University Press, New York, NY, USA, 2010. 
[26] I. Zarudi and L. C. Zhang, "A revisit to some wheel-workpiece interaction problems in surface grinding," International Journal of Machine Tools and Manufacture, vol. 42, no. 8, pp. 905-913, 2002.

[27] M. R. Spiegel, Handbook of Mathematical Formulas, SchaumMcGraw Hill, New York, NY, USA, 1968.

[28] N. N. Lebedev, Special Functions and Their Applications, Dover, New York, NY, USA, 1972.

[29] G. E. Andrews, R. Askey, and R. Roy, Special Functions, Cambridge University Press, Cambridge, UK, 1999. 


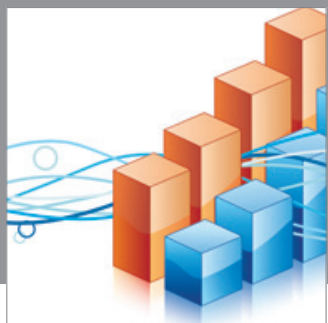

Advances in

Operations Research

mansans

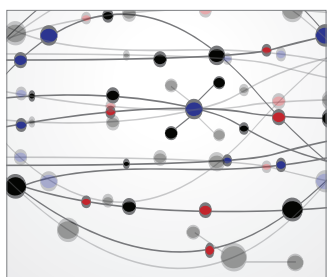

The Scientific World Journal
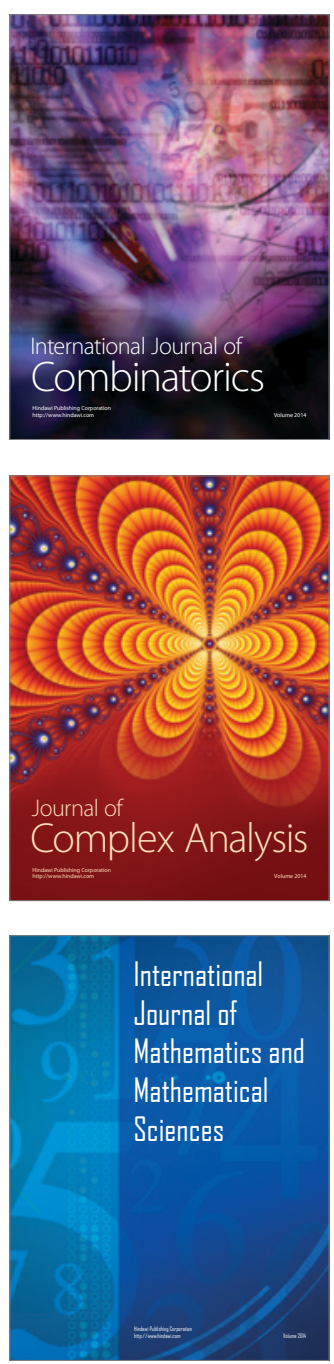
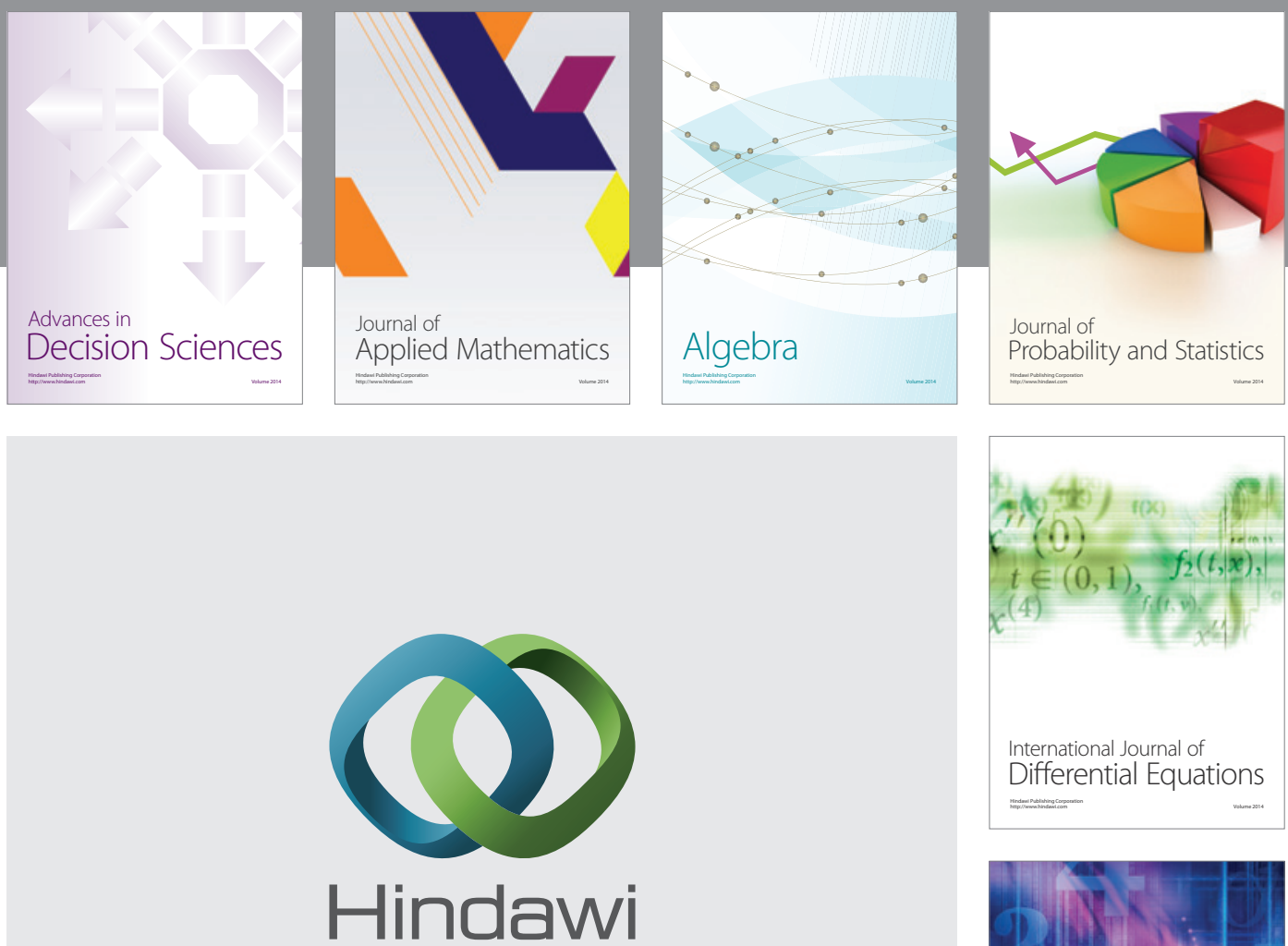

Submit your manuscripts at http://www.hindawi.com
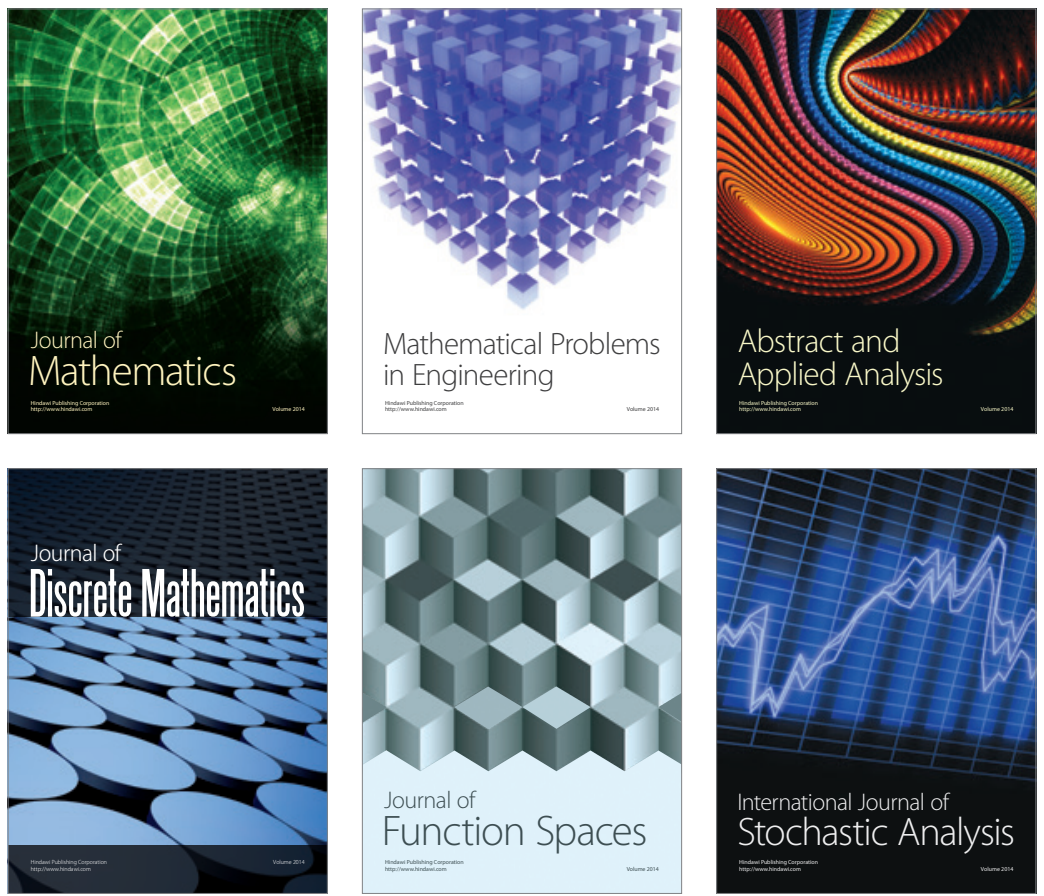

Journal of

Function Spaces

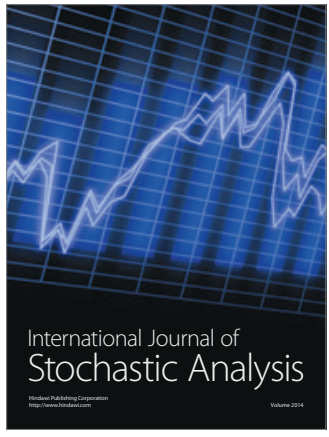

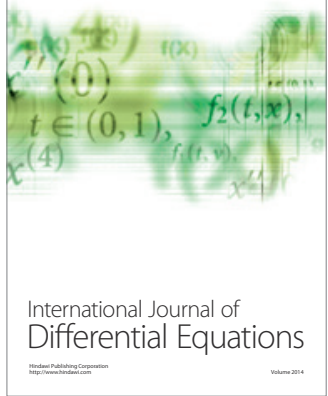
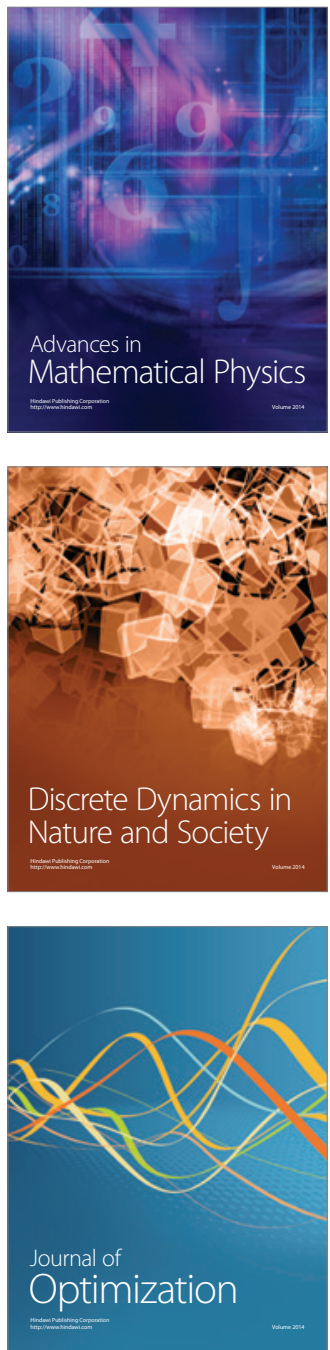